\title{
Adult T-cell Leukemia/Lymphoma as a Methotrexate-associated Lymphoproliferative Disorder in a Patient with Rheumatoid Arthritis
}

\author{
Ichiro Takajo $^{1,2}$, Kunihiko Umekita ${ }^{1}$, Yoshihiko Ikei $^{2}$, Koichi Oshima $^{3}$ and Akihiko Okayama ${ }^{1}$
}

\begin{abstract}
:
The patient was a 74-year-old Japanese woman with rheumatoid arthritis (RA) who developed generalized lymphadenopathy with elevated levels of lactase dehydrogenase (LD), and soluble IL-2 receptor (sIL-2R). She was found to be positive for anti-human T-cell leukemia virus type 1 (HTLV-1) antibodies. Her symptoms and laboratory abnormalities spontaneously regressed after the cessation of methotrexate (MTX), suggesting that she had an MTX-associated lymphoproliferative disorder; however, her lymphadenopathy appeared again approximately 14 months later with LD and sIL-2R elevation. A histopathological analysis and Southern blotting of a lymph node biopsy specimen for HTLV-1 provirus supported the diagnosis of adult Tcell leukemia/lymphoma (ATL) (lymphoma type). These data confirmed that an HTLV-1 positive RA patient may develop ATL.
\end{abstract}

Key words: rheumatoid arthritis, human T-lymphotropic virus type 1, adult T-cell leukemia/lymphoma, methotrexate-associated lymphoproliferative disorder

(Intern Med 57: 2071-2075, 2018)

(DOI: 10.2169/internalmedicine.0308-17)

\section{Introduction}

Patients with rheumatoid arthritis (RA) may present with a wide spectrum of lymphoid proliferation and lymphomas, which together are named lymphoproliferative disorders (LPD). The standardized incidence ratio (SIR) for lymphoma in Japanese RA patients is reported to be significantly high $(1,2)$. B-cell phenotype has been reported to occur in as much as $80 \%$ of LPDs (3). The most common histology of methotrexate (MTX)-LPD found in Japanese RA patients was diffuse large B-cell lymphoma; its frequency was as high as $-60 \%$ of reported cases (3). The risk of developing LPD was reported to be increased when RA patients were treated with MTX (4). Epstein-Barr virus (EBV) infection has been considered to be involved in the pathogenesis of LPD. A systematic review showed that EBVinduced LPD in RA patients is commonly seen in Asian individuals (5). EBV was detected in $60 \%$ of Japanese RA pa- tients with MTX-associated LPD (6). Discontinuation of MTX treatment may result in the spontaneous regression of MTX-associated $\operatorname{LPD}(7,8)$. Thus, immunosuppression caused by MTX has been suspected to be associated with the development of EBV-related B-cell LPD in RA patients.

Human T-cell leukemia virus type 1 (HTLV-1) is the causative agent of the T-cell malignancy adult T-cell leukemia/lymphoma (ATL) (9). The acute or lymphoma types of ATL are aggressive diseases and the prognosis has been reported to be poor, even when they were treated with advanced chemotherapy (10). HTLV-1 is endemic in Japan, where there are an estimated 800,000 HTLV-1 carriers (11). Because women have a higher prevalence of HTLV-1 infection (11), it is reasonable to assume that a relatively high number of Japanese RA patients are infected with HTLV-1. Thus far, only a small number of patients with MTX-LPD have been reported to have the T-cell phenotype and no cases of ATL have been reported as MTX-LPD $(3,8)$. Thus, whether or not HTLV-1-positive RA patients are at greater

${ }^{1}$ Department of Rheumatology, Infectious Diseases and Laboratory Medicine, Internal Medicine, Faculty of Medicine, University of Miyazaki, Japan, ${ }^{2}$ Youkikai Ikei Hospital, Japan and ${ }^{3}$ Department of Pathology, Kurume University, Japan

Received: October 4, 2017; Accepted: November 29, 2017; Advance Publication by J-STAGE: February 28, 2018

Correspondence to Dr. Kunihiko Umekita, kunihiko_umekita@med.miyazaki-u.ac.jp 


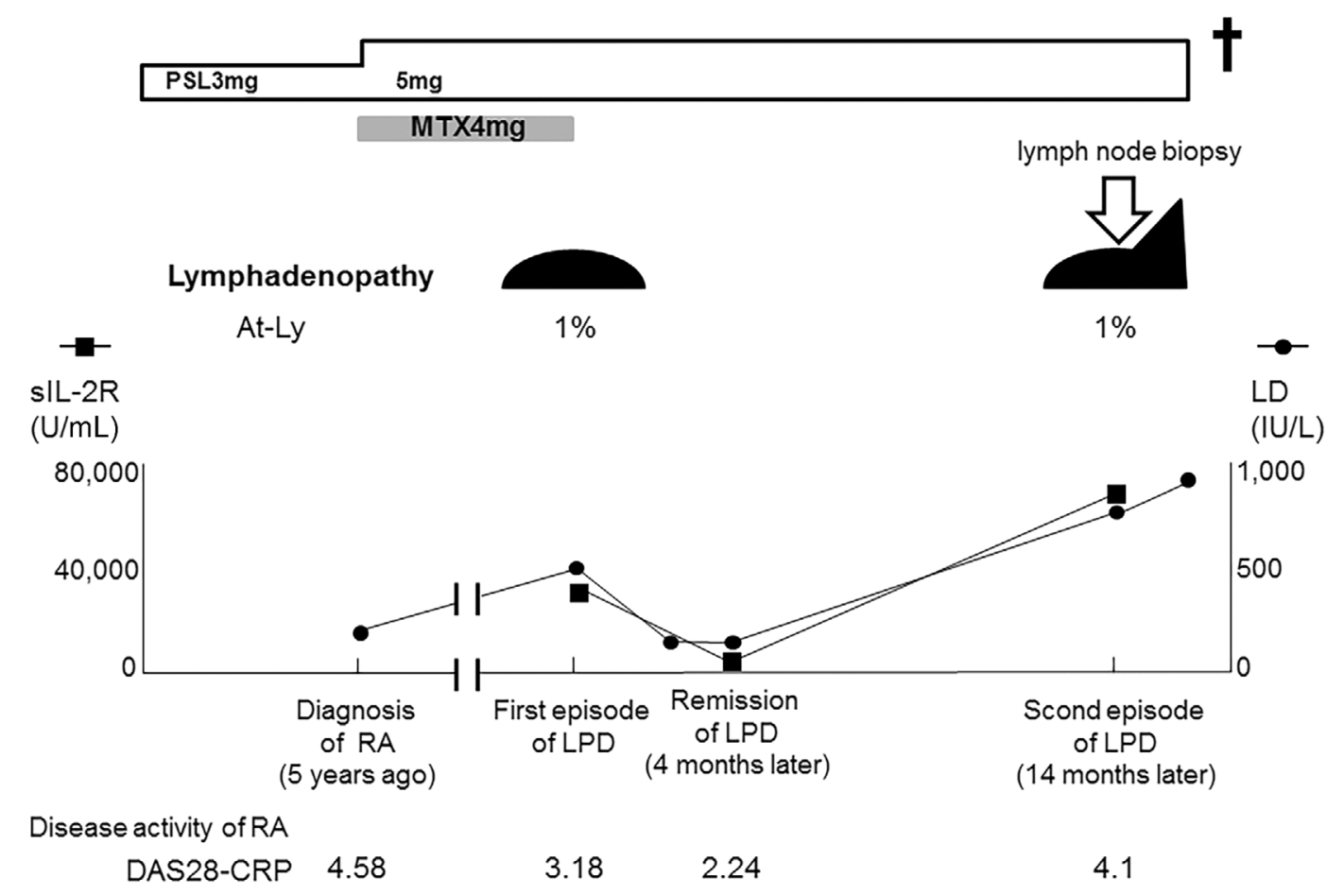

Figure 1. The clinical course. PSL: prednisolone, MTX: methotrexate, LD: lactate dehydrogenase, At-Ly: atypical lymphocytes, sIL-2R: soluble interleukin 2 receptor, RA: rheumatoid arthritis, LPD: related lymphoproliferative disease, DAS28-CRP: disease activity score 28 joints-C-reactive protein

risk of ATL - especially when they receive anti-rheumatic treatment including MTX - is an important question.

We report the case of an RA patient, who developed generalized lymphadenopathy that showed spontaneous regression after the withdrawal of MTX, suggesting that she had an MTX-associated LPD. She was found to be positive for HTLV-1 and developed ATL (lymphoma type) 14 months later. It was not clear whether the first episode of MTXassociated LPD occurred due to ATL because no biopsy was performed at the time; however, this case raised the question as to whether T-cell LPD can occur in HTLV-1-positive RA patients who are treated with MTX.

\section{Case Report}

A 74-year-old Japanese woman presented to our hospital with poly-arthritis. She tested positive for anti-citrullinated protein antibodies and was diagnosed with seropositive RA based on the 2010 American College of Rheumatology/ European League Against Rheumatism criteria. Her disease activity score 28 joints-C-reactive protein (DAS28-CRP) was 4.58, suggesting moderate disease activity. We treated her moderate RA with MTX (4 mg per week) and prednisolone (PSL; 5 mg per day).

Her condition remained stable for 5 years; however, she complained of anorexia and lymph node enlargement was recognized in the left cervical region. Her serum levels of lactase dehydrogenase (LD, $433 \mathrm{IU} / \mathrm{L}$ ) and CRP (2.14 mg/ dL) were elevated. The serum level of soluble IL-2 receptor (sIL-2R) was markedly elevated $(34,881 \mathrm{U} / \mathrm{mL})$ and a small number of atypical lymphocytes were present in the peripheral blood (Fig. 1). Although typical ATL cells were not found, HTLV-1 antibodies were detected. Computed tomography revealed generalized lymphadenopathy including the mediastinum and abdomen (Fig. 2). MTX treatment was immediately discontinued, and her lymph node enlargement improved. Her serum LD and sIL-2R levels were found to have decreased to normal levels (161 IU/L and $663 \mathrm{U} / \mathrm{mL}$, respectively) at four months after MTX withdrawal. Because MTX withdrawal induced spontaneous regression, she was diagnosed with MTX-associated LPD; however, lymph node biopsy was not performed. Thereafter, her RA was well controlled with a low dose of PSL (3 mg/day).

At fourteen months after the initial event of LPD, she again complained of anorexia and exhibited lymph node enlargement in the left cervical region. On CT, generalised lymphadenopathy, which included the mediastinum and abdomen, was found to be worse than that observed during the first episode of LPD (data not shown). The patient's serum LD (729 IU/L) and sIL-2R levels $(73,208 \mathrm{U} / \mathrm{mL})$ were markedly elevated. The peripheral blood again showed a small number of atypical lymphocytes without typical ATL cells. We performed a cervical lymph node biopsy. A pathological analysis revealed the diffuse proliferation of mediumsized abnormal lymphocytes, suggesting malignant lymphoma (Fig. 3). On immunostaining, the surface marker profile of the lymphocytes with atypia in the lymph node specimen was CD20(-), CD3(+), CD4(+), CD8(-), and CD30(-). The Epstein-Barr encoding region was negative upon in situ hybridization; this was compatible with a T-cell phenotype 

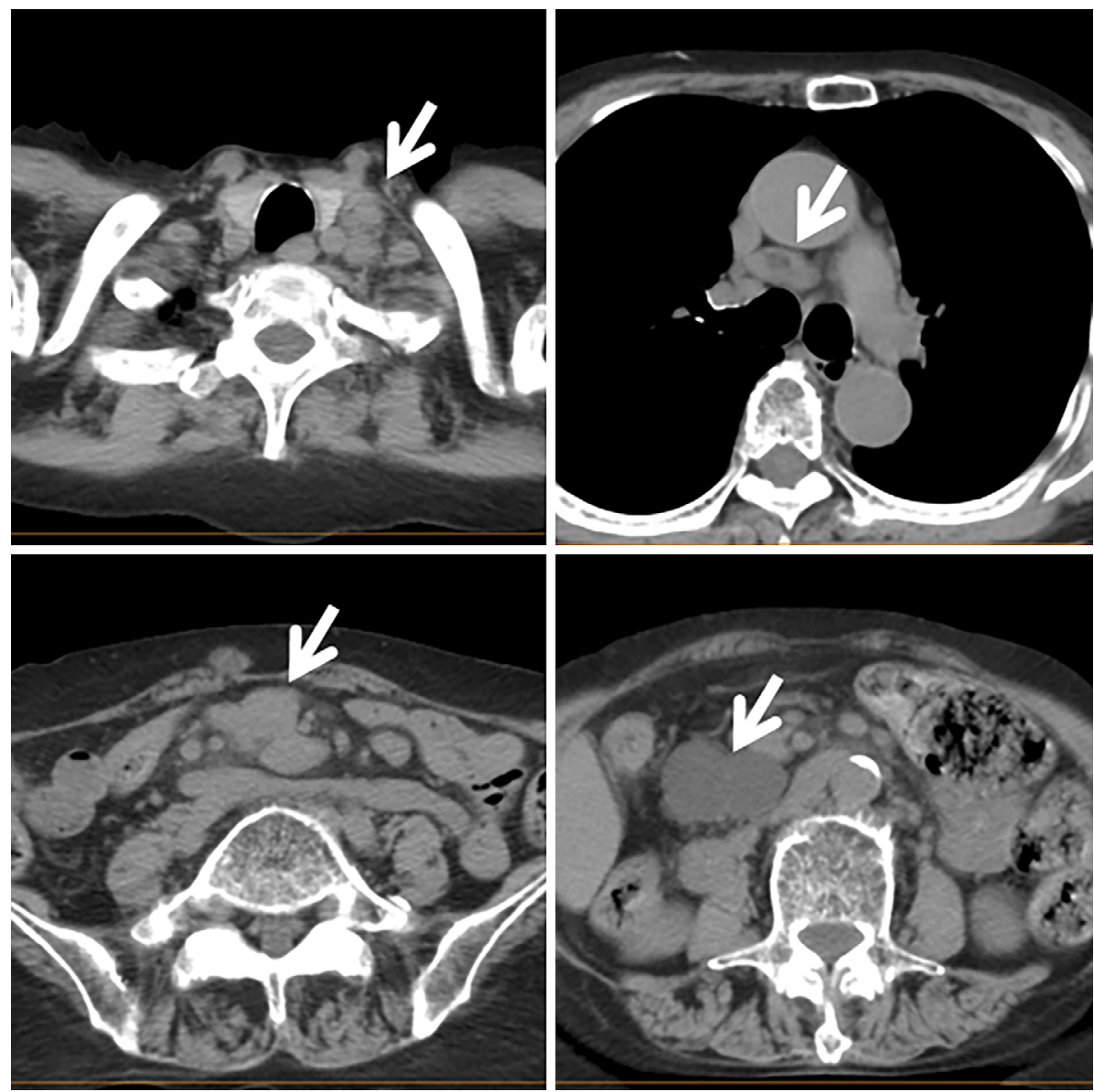

Figure 2. Computed tomography at the levels of the cervix, mediastinum, and abdomen. The enlarged lymph nodes are indicated with an arrowhead.

without EBV infection. Southern blotting of the lymph node specimen for HTLV-1 provirus showed a monoclonal proliferation of HTLV-1-infected cells (Fig. 4). She was referred to a hematologist and was diagnosed with ATL (lymphoma type) based on Shimoyama's criteria (12). Her general condition did not allow us to perform chemotherapy for ATL and she died within a month.

\section{Discussion}

It has been realized that RA patients have a higher risk of developing Hodgkin lymphoma and non-Hodgkin lymphoma (13). In addition, immunosuppressive treatments, including MTX, have been reported to increase the risk of LPD. Spontaneous regression after the withdrawal of MTX treatment has been reported, especially in RA patients with EBV-associated $\operatorname{LPD}(3,4,8)$. Thus, the reactivation of EBV in RA patients with LPD may be induced by immunosuppressive treatment, including MTX (8).

In contrast, ATL is a malignancy of mature T-cells caused by HTLV-1 infection (9). The incidence of ATL among HTLV-1 carriers has been reported to be one in 1,000 person-years (10). There have been several case reports of ATL in patients with rheumatic diseases. Nakamura et al. reported a case of ATL developing in an RA patient who was treated with MTX and tocilizumab for 7 years (14). The complete remission of ATL was achieved with a standard therapeutic regimen, which suggested a preferable clinical course. Moreover, a case was reported in which a spondyloarthritis patient treated with the anti-TNF adalimumab developed ATL (15). This patient was reported to have had a history of oral immunosuppressant treatment; however, it was not clear whether MTX was prescribed. We recently experienced a case in which ATL (chronic type) developed in an HTLV-1 positive RA patient (16). The patient was treated with MTX and infliximab when she developed ATL. After the discontinuation of MTX and infliximab, her ATL showed spontaneous remission. These cases raised the question as to whether ATL occurs as T-cell LPD in HTLV-1positive patients with rheumatic diseases-especially RAwhen they are treated with immunosuppressive agents, including MTX and/or biologic agents.

In the present study, we experienced a case of MTX-LPD followed by the occurrence of overt ATL in an HTLV-1positive RA patient. The question was whether or not the first episode of LPD in this case was ATL. The first episode might have been EBV-associated B-cell MTX-LPD, because this type of LPD is known to frequently regress upon the withdrawal of MTX (8). If this was the case, two different types of LPD occurred in the same patient within a rela- 


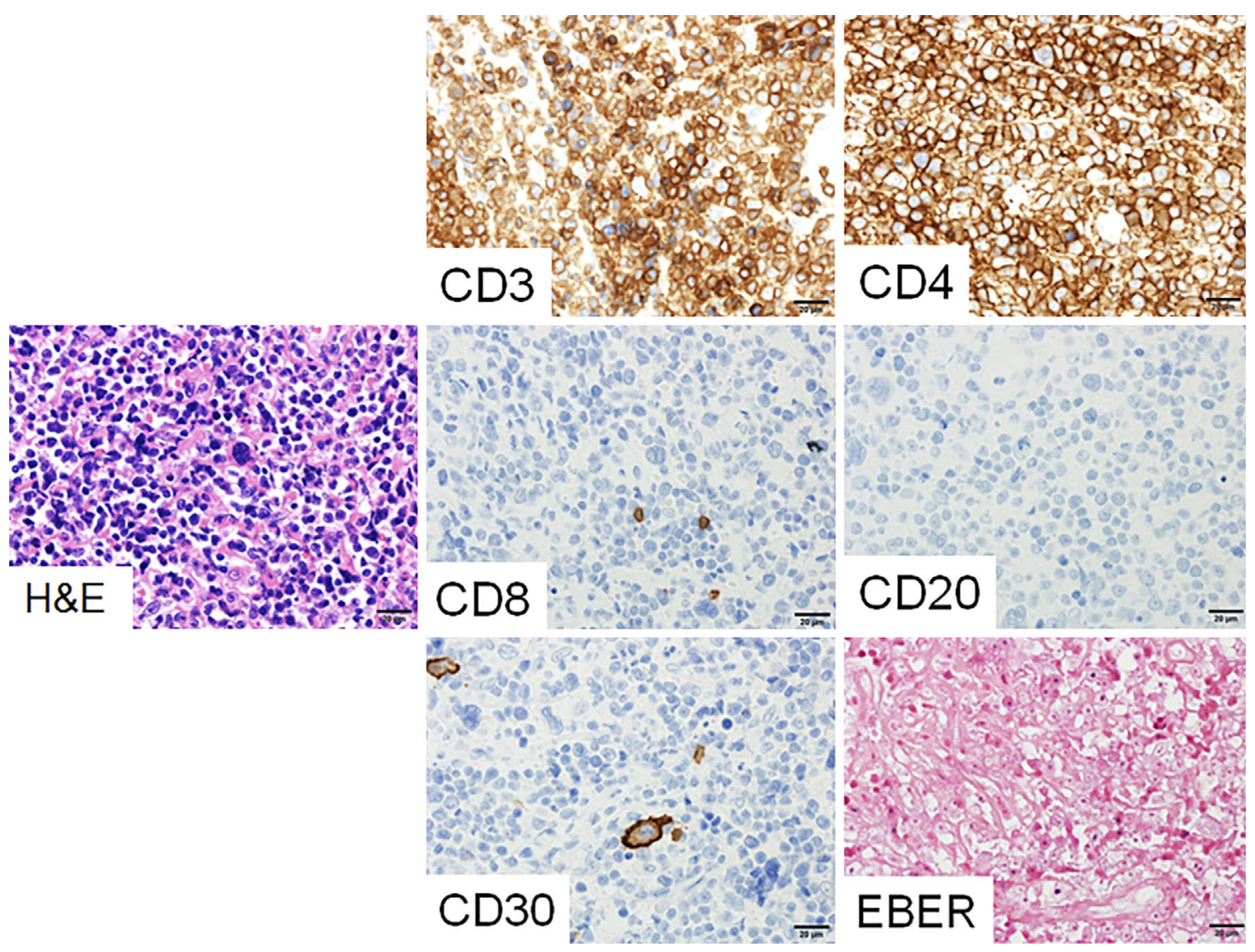

Figure 3. Immuno-histological and in situ hybridization analyses of the lymph node biopsy specimen. The abnormal lymphocytes were CD3 (+) CD4 (+) CD8 (+) CD20 (-) CD30 (-) and EBER (-). The histopathological diagnosis was compatible with adult T-cell leukemia lymphoma. H\&E: Hematoxylin and Eosin staining, CD: cluster of differentiation, EBER: Epstein-Barr encoding region

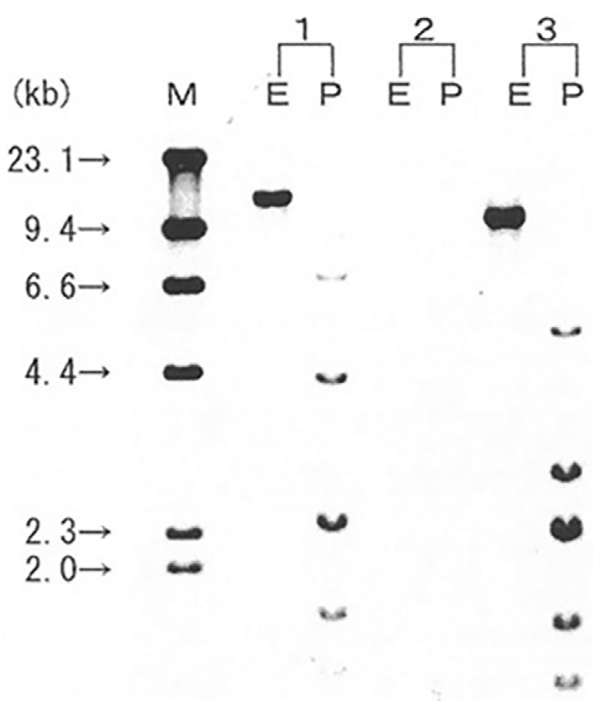

Figure 4. Southern blotting of the lymph node biopsy specimen to detect HTLV-1 proviral DNA. Southern blotting revealed the monoclonal integration of HTLV-1 proviral DNA (Special Reference Laboratories). 1, positive control; 2, negative control; 3 , patient specimen; $M$, size marker ( $\lambda$ DNA/HindIII). Restriction enzyme (E: Eco RI, P: Pst I). tively short period. However, the median sIL-2R level in patients with diffuse large B-cell lymphoma is reported to be $1,473 \mathrm{U} / \mathrm{mL}$, while that of ATL (lymphoma type) is reported to be as high as $34,620 \mathrm{U} / \mathrm{mL}(17,18)$. The marked elevation of sIL-2R in the present case not only in the second episode (ATL), but also in the first episode of LPD, supported the possibility that the first episode was also ATL.

A high HTLV-1 proviral DNA load and the clonal expansion of HTLV-1-infected cells in asymptomatic HTLV-1 carriers have been considered major risk factors for the development ATL $(19,20)$. The incidence of ATL has been reported to increase upon immunosuppressive treatment. HTLV-1-positive patients who received liver transplantation followed by immunosuppressive treatment were reported to have a high risk of developing ATL (21). A neurological disease, HTLV-1-associated myelopathy, was also reported to have developed in a patient undergoing living-donor liver transplantation (22). It is possible that the active expansion of HTLV-1-infected T-cells may occur in HTLV-1-positive patients who are treated with immunosuppressive agents. If the first episode of the present case was less aggressive ATL, it is possible that treatment with MTX caused immunosuppression and was involved in the development of ATL. The withdrawal of MTX may have induced the recovery of the immune system, resulting in the spontaneous re- 
gression of ATL; however, the patient in the present case developed aggressive ATL within one year. A similar situation, in which aggressive lymphoma develops after the initial regression of LPD, is commonly observed in patients with EBV-associated MTX-LPD (3).

One of the limitations of the present report is that no biopsy was performed during the first episode of lymphadenopathy. Thus, the nature of MTX-LPD, including the pathology, surface phenotype, and association with EBV or HTLV1 , was not determined in the first episode of MTX-LPD.

In conclusion, the present report confirmed that an HTLV1-positive RA patient may develop ATL. In addition, the current case indicated the possibility that less aggressive ATL occurs as MTX-LPD, which only regresses upon the withdrawal of MTX. If this is true, then HTLV-1-positive RA patients treated with MTX should be carefully followed to allow for the early diagnosis of ATL. Further studies, with large numbers of HTLV-1-positive RA patients, will be necessary to resolve the clinical question as to whether HTLV-1 infection causes ATL as a T-cell LPD in RA patients and whether MTX treatment is a risk factor for ATL in such patients.

Written informed consent for the publication of this report was obtained from the patient's son by the author.

\section{The authors state that they have no Conflict of Interest (COI).}

\section{Financial Support}

This work was partly supported by the Japan Agency for Medical Research and Development.

\section{Acknowledgement}

We thank Dr. Yoko Kubuki and Dr. Takuro Kameda of Youkika-Ikei Hospital and Dr. Kouki Tokuda of Kobayashi city hospital for their clinical assistance.

\section{References}

1. Hashimoto A, Chiba $\mathrm{N}$, Tsuno $\mathrm{H}$, et al. Incidence of malignancy and the risk of lymphoma in Japanese patients with rheumatoid arthritis compared to the general population. J Rheumatol 42: 564571, 2015.

2. Yamada T, Nakajima A, Inoue E, et al. Incidence of malignancy in Japanese patients with rheumatoid arthritis. Rheumatol Int 31: 1487-1492, 2011

3. Hoshida Y, Xu JX, Fujita S, et al. Lymphoproliferative disorders in rheumatoid arthritis: clinicopathological analysis of 76 cases in relation to methotrexate medication. J Rheumatol 34: 322-331, 2007.

4. Berti A, Felicetti M, Peccatori S, et al. EBV-induced lymphoproliferative disorders in rheumatic patients: a systematic review of the literature. Joint Bone Spine 85: 35-40, 2018.

5. Ichikawa A, Arakawa F, Kiyasu J, et al. Methotrexate/iatrogenic lymphoproliferative disorders in rheumatoid arthritis: histology, Epstein-Barr virus, and clonality are important predictors of disease progression and regression. Eur J Haematol 91: 20-28, 2013.

6. Kameda T, Dobashi H, Miyatake N, et al. Association of higher methotrexate dose with lymphoproliferative disease onset in rheu- matoid arthritis patients. Arthritis Care Res (Hoboken) 66: 13021309, 2014.

7. Niitsu N, Okamoto M, Nakamine H, Hirano M. Clinicopathologic correlations of diffuse large B-cell lymphoma in rheumatoid arthritis patients treated with methotrexate. Cancer Sci 101: 1309-1313, 2010.

8. Miyazaki T, Fujimaki K, Shirasugi Y, et al. Remission of lymphoma after withdrawal of methotrexate in rheumatoid arthritis: relationship with type of latent Epstein-Barr virus infection. Am J Hematol 82: 1106-1109, 2007.

9. Matsuoka M. Human T-cell leukemia virus type I and adult T-cell leukemia. Oncogene 22: 5131-5140, 2003.

10. Tsukasaki K, Tobinai K. Human T-cell lymphotropic virus type Iassociated adult T-cell leukemia-lymphoma: new directions in clinical research. Clin Cancer Res 20: 5217-5225, 2014.

11. Satake M, Yamaguchi K, Tadokoro K. Current prevalence of HTLV-1 in Japan as determined by screening of blood donors. J Med Virol 84: 327-335, 2012.

12. Shimoyama M. Diagnostic criteria and classification of clinical subtypes of adult T-cell leukaemia-lymphoma. A report from the Lymphoma Study Group (1984-87). Br J Haematol 79: 428-437, 1991.

13. Harigai M, Nanki T, Koike R, et al. Risk for malignancy in rheumatoid arthritis patients treated with biological disease-modifying anti rheumatic drugs compared to the general population: a nationwide cohort study in Japan. Mod Rheumatol 26: 642-650, 2016.

14. Nakamura H, Ueki Y, Saito S, et al. Development of adult T-cell leukemia in a patient with rheumatoid arthritis treated with tocilizumab. Intern Med 52: 1983-1986, 2013.

15. Bittencourt AL, Oliveira PD, Bittencourt VG, Carvalho EM, Farre L. Adult T-cell leukemia/lymphoma triggered by adalimumab. J Clin Virol 58: 494-496, 2013.

16. Hashiba Y, Hidaka T, Umekita K, et al. Remission of chronic type ATL in a patient with rheumatoid arthritis after withdrawing methotrexate and anti-TNF $\alpha$ biologic combination therapy; a case report. Modern Rheum Case Reports 2: 9-13, 2018.

17. Goto $H$, Tsurumi $H$, Takemura $M$, et al. Serum-soluble interleukin-2 receptor (sIL-2R) level determines clinical outcome in patients with aggressive non-Hodgkin's lymphoma: in combination with the International Prognostic Index. J Cancer Res Clin Oncol 131: 73-79, 2005.

18. Kamihira S, Atogami S, Sohda H, Momita S, Yamada Y, Tomonaga M. Significance of soluble interleukin-2 receptor levels for evaluation of the progression of adult T-cell leukemia. Cancer 73: 2753-2757, 1994.

19. Iwanaga M, Watanabe T, Utsunomiya A, et al. Human T-cell leukemia virus type I (HTLV-1) proviral load and disease progression in asymptomatic HTLV-1 carriers: a nationwide prospective study in Japan. Blood 116: 1211-1219, 2010.

20. Okayama A, Stuver S, Matsuoka M, et al. Role of HTLV-1 proviral DNA load and clonality in the development of adult T-cell leukemia/lymphoma in asymptomatic carriers. Int J Cancer 110: 621625, 2004.

21. Kawano N, Shimoda K, Ishikawa F, et al. Adult T-cell leukemia development from a human T-cell leukemia virus type I carrier after a living-donor liver transplantation. Transplantation 82: 840843, 2006.

22. Soyama A, Eguchi S, Takatsuki M, et al. Human T-cell leukemia virus type I-associated myelopathy following living-donor liver transpl. Liver Transpl 14: 647-650, 2008.

The Internal Medicine is an Open Access article distributed under the Creative Commons Attribution-NonCommercial-NoDerivatives 4.0 International License. To view the details of this license, please visit (https://creativecommons.org/licenses/ by-nc-nd/4.0/).

(C) 2018 The Japanese Society of Internal Medicine Intern Med 57: 2071-2075, 2018 